\title{
Dynamics analysis of an online gambling spreading model on scale-free networks
}

\author{
Yu Kong ${ }^{1}$, Tao Li ${ }^{1 *} \mathbb{D}$, Yuanmei Wang ${ }^{1}$, Xinming Cheng ${ }^{2}$, He Wang ${ }^{1}$ and Yangmei Lei ${ }^{1}$
}

${ }^{\text {*Correspondence: }}$

taohust2008@163.com

ischool of Electronics and Information, Yangtze University, Jingzhou, 434023, P.R. China Full list of author information is available at the end of the article

\begin{abstract}
Nowadays, online gambling has a great negative impact on the society. In order to study the effect of people's psychological factors, anti-gambling policy, and social network topology on online gambling dynamics, a new SHGD (susceptible-hesitator-gambler-disclaimer) online gambling spreading model is proposed on scale-free networks. The spreading dynamics of online gambling is studied. The basic reproductive number $R_{0}$ is got and analyzed. The basic reproductive number $R_{0}$ is related to anti-gambling policy and the network topology. Then, gambling-free equilibrium $E_{0}$ and gambling-prevailing equilibrium $E_{+}$are obtained. The global stability of $E_{0}$ is analyzed. The global attractivity of $E_{+}$and the persistence of online gambling phenomenon are studied. Finally, the theoretical results are verified by some simulations.
\end{abstract}

Keywords: SHGD model; Heterogeneity; Psychological factors; Anti-gambling policy; Stability; Persistence

\section{Introduction}

Online gambling has emerged with the wide use of network technology. Compared with traditional gambling, the online gambling is stronger interaction, higher concealment, and more difficult to control [1]. Obviously, online gambling spreads more easily and widely than traditional gambling. The widespread spread of online gambling phenomenon has a huge negative impact on society [2-4].

How to control the phenomenon of online gambling is very important. Some scholars have studied the phenomenon of online gambling from different aspects [5-9]. King and Barak [10] studied the characteristics of online gambling such as attraction, convenience, and reasons why people participate in gambling. Dickson-Gillespie et al. [11] found that effective educational programs, media campaigns, and public policy would be good for quitting gambling. In addition, we should note the network spread characteristic of online gambling [12]. So, it is important for us to study the spreading dynamics of online gambling. Through the study of online gambling dynamics, we can comprehensively and systematically learn about the spreading mechanism and influence factors, which is more helpful to control the spread of online gambling.

Research on spreading dynamics of online gambling is relatively rare at present. There are some results in information spreading dynamics and disease spreading dynamics [13-

(c) The Author(s) 2021. This article is licensed under a Creative Commons Attribution 4.0 International License, which permits use sharing, adaptation, distribution and reproduction in any medium or format, as long as you give appropriate credit to the original author(s) and the source, provide a link to the Creative Commons licence, and indicate if changes were made. The images or other third party material in this article are included in the article's Creative Commons licence, unless indicated otherwise in a credit line to the material. If material is not included in the article's Creative Commons licence and your intended use is not permitted by statutory regulation or exceeds the permitted use, you will need to obtain permission directly from the copyright holder. To view a copy of this licence, visit http://creativecommons.org/licenses/by/4.0/. 
18]. Liu et al. [19] studied the spread dynamics of word-of-mouth. Wang et al. [20] proposed a network epidemic model for waterborne diseases spread and considered both indirect environment-to-human and direct human-to-human transmission routes. King et al. [21] established a two-way model, studied the influence of some background factors to gambling spread. However, in the research works mentioned above, the persistence of online gambling phenomenon and the global attractivity of online gambling equilibrium are not studied. Meanwhile, some researchers found that the scale-free property is an important property of social networks [22, 23]. Obviously, the spread networks of online gambling are based on social networks. So, based on scale-free networks, we study the dynamics of online gambling in the paper. Taking into account people's psychological factors, anti-gambling policy, we present a new comprehensively SHGD (susceptible-hesitatorgambler-disclaimer) online gambling spreading model.

The rest of the paper is as follows: The SHGD online gambling spreading model is presented and described in Sect. 2 . The basic reproductive number $R_{0}$, gambling-free equilibrium $E_{0}$, and gambling-prevailing equilibrium $E_{+}$are derived in Sect. 3 . Then, the stability of $E_{0}$, the global attraction of $E_{+}$, and the persistence of online gambling phenomenon are studied. Some simulations are shown in Sect. 4. We conclude the paper in Sect. 5.

\section{Model formulation}

We present a new SHGD (susceptible-hesitator-gambler-disclaimer) online gambling spreading model. The model has the spread sketch in Fig. 1. In the model, nodes are used to stand for individuals, and edges are used to stand for the relationships between individuals. The whole crowd is divided into four different classes, namely susceptible $(S)$, hesitator $(H)$, gambler $(G)$, and disclaimer $(D)$. $S$ nodes represent individuals who are not involved in gambling currently and can be influenced by the online gambling behavior; $H$ nodes represent individuals who know the phenomenon of online gambling and hesitate whether to participate in online gambling and can spread online gambling behavior; $G$ nodes represent individuals who take part in online gambling and can spread the online gambling behavior; $D$ nodes represent the individuals who have given up gambling.

The transitions of these states are as follows:

(1) When a susceptible individual connects with a hesitator or a gambler, he or she can be influenced and become a hesitator with probability $\beta_{1}$ or $\beta_{2}$, respectively.

(2) The parameter $\varepsilon$ represents the probability that a hesitator becomes a susceptible individual. The parameter $\eta$ represents the probability that a hesitator becomes a gambler. The parameter $\chi$ indicates the influence degree of the anti-gambling policy

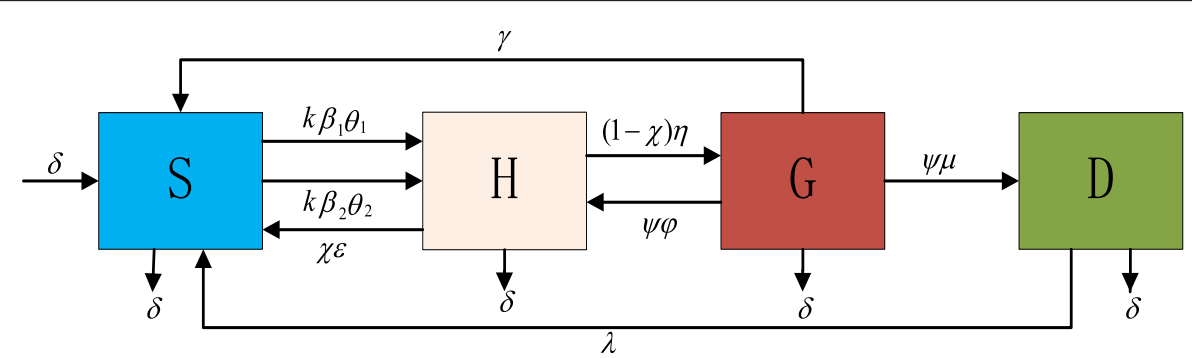

Figure 1 The flow diagram of the SHGD model 
to the hesitator. Considering the influence degree of the anti-gambling policy, the hesitator will become a susceptible individual with the probability $\chi \varepsilon$, in contrast, a gambler with the probability $(1-\chi) \eta$.

(3) The parameter $\varphi$ represents the probability that a gambler becomes a hesitator. The parameter $\mu$ represents the probability that a gambler becomes a disclaimer. The parameter $\psi$ represents the influence degree of the anti-gambling policy to the gambler. Considering the influence degree of the anti-gambling policy, a gambler will become a hesitator or a disclaimer with the probability $\psi \varphi$ or $\psi \mu$, respectively. A gambler will become a susceptible individual with the probability $\gamma$ when he or she loses interest in online gambling.

(4) Because of the psychological factors of the disclaimer, such as forgetting and so on, the disclaimer will become a susceptible individual with the probability $\lambda$.

(5) The probability $\delta$ is the register rate and logout rate. Assume newcomers are susceptible individuals.

We define $S_{k}(t), H_{k}(t), G_{k}(t), D_{k}(t)$ as the relative densities of susceptible, hesitator, gambler, and disclaimer nodes at time $t$, respectively, where $k$ is the node degree. According to the above description and assumption, we can get the SHGD model as follows:

$$
\left\{\begin{aligned}
\frac{d S_{k}(t)}{d t}= & \delta+\lambda D_{k}(t)+\gamma G_{k}(t)+\chi \varepsilon H_{k}(t)-k \beta_{1} \theta_{1}(t) S_{k}(t)-k \beta_{2} \theta_{2}(t) S_{k}(t)-\delta S_{k}(t), \\
\frac{d H_{k}(t)}{d t}= & k \beta_{1} \theta_{1}(t) S_{k}(t)+k \beta_{2} \theta_{2}(t) S_{k}(t)+\psi \varphi G_{k}(t) \\
& -(1-\chi) \eta H_{k}(t)-\chi \varepsilon H_{k}(t)-\delta H_{k}(t), \\
\frac{d G_{k}(t)}{d t}= & (1-\chi) \eta H_{k}(t)-\psi \mu G_{k}(t)-\gamma G_{k}(t)-\psi \varphi G_{k}(t)-\delta G_{k}(t), \\
\frac{d D_{k}(t)}{d t}= & \psi \mu G_{k}(t)-\lambda D_{k}(t)-\delta D_{k}(t),
\end{aligned}\right.
$$

where $\theta_{1}(t)$ is the probability of linking to a hesitator at time $t$ and satisfies

$$
\theta_{1}(t)=\frac{\sum_{k} k Q(k) H_{k}(t)}{\sum_{k} s Q(s)}=\frac{1}{\langle k\rangle} \sum_{k} k Q(k) H_{k}(t)
$$

where $\theta_{2}(t)$ is the probability of linking to a gambler at time $t$ and satisfies

$$
\theta_{2}(t)=\frac{\sum_{k} k Q(k) G_{k}(t)}{\sum_{k} s Q(s)}=\frac{1}{\langle k\rangle} \sum_{k} k Q(k) G_{k}(t)
$$

Here, $\langle k\rangle$ represents the average degree values in the network, and $Q(k)$ represents the degree distribution. $H(t)=\sum_{k} Q(k) H_{k}(t)$ is the density of the hesitator, and $G(t)=$ $\sum_{k} Q(k) G_{k}(t)$ is the density of the gambler. We make $\rho(t)=\beta_{1} \theta_{1}+\beta_{2} \theta_{2}$. And according to system (1), we can get

$$
\left\{\begin{array}{l}
\frac{d S_{k}(t)}{d t}=\delta+\lambda D_{k}(t)+\gamma G_{k}(t)+\chi \varepsilon H_{k}(t)-(k \rho(t)+\delta) S_{k}(t), \\
\frac{d H_{k}(t)}{d t}=k \rho(t) S_{k}(t)+\psi \varphi G_{k}(t)-((1-\chi) \eta+\chi \varepsilon+\delta) H_{k}(t), \\
\frac{d G_{k}(t)}{d t}=(1-\chi) \eta H_{k}(t)-(\psi \mu+\gamma+\psi \varphi+\delta) G_{k}(t), \\
\frac{d D_{k}(t)}{d t}=\psi \mu G_{k}(t)-(\lambda+\delta) D_{k}(t) .
\end{array}\right.
$$


According to the normalization conditions, we can know $S_{k}(t)+H_{k}(t)+G_{k}(t)+D_{k}(t)=1$. The initial conditions for the system are as follows:

$$
\left\{\begin{array}{l}
0 \leq S_{k}(0), H_{k}(0), G_{k}(0), D_{k}(0) \leq 1, \\
\rho(0)>0 .
\end{array}\right.
$$

\section{The basic reproductive number and equilibriums}

In the section, we analyze the properties of the SHGD online gambling spreading model.

Theorem 1 According to system (4), the basic reproductive number is defined as follows:

$$
R_{0}=\frac{\left\langle k^{2}\right\rangle}{\langle k\rangle} \frac{(\psi \mu+\gamma+\psi \varphi+\delta) \beta_{1}+\eta(1-\chi) \beta_{2}}{(\psi \mu+\gamma+\delta)((1-\chi) \eta+\chi \varepsilon+\delta)+\psi \varphi(\chi \varepsilon+\delta)} .
$$

Consider system (4), we can get:

(1) There is a gambling-free equilibrium $E_{0}(1,0,0,0)$ when $R_{0}<1$.

(2) There is a unique gambling-prevailing equilibrium $E_{+}\left(S_{k}^{*}, H_{k}^{*}, G_{k}^{*}, D_{k}^{*}\right)$ when $R_{0}>1$.

Proof It can be easy to find that system (4) satisfies $S_{k}(t)=1-H_{k}(t)-G_{k}(t)-D_{k}(t)$. According to system (4), we can get

$$
\left\{\begin{array}{l}
\frac{d H_{k}(t)}{d t}=k \rho(t)\left(1-H_{k}(t)-G_{k}(t)-D_{k}(t)\right)+\psi \varphi G_{k}(t)-((1-\chi) \eta+\chi \varepsilon+\delta) H_{k}(t) \\
\frac{d G_{k}(t)}{d t}=(1-\chi) \eta H_{k}(t)-(\psi \mu+\gamma+\psi \varphi+\delta) G_{k}(t) \\
\frac{d D_{k}(t)}{d t}=\psi \mu G_{k}(t)-(\lambda+\delta) D_{k}(t)
\end{array}\right.
$$

Obviously, there is a gambling-free equilibrium $E_{0}=\{(0,0,0)\}_{k}$ in system (7). By using the next generation matrix method [24], system (7) can be written

$$
\frac{d x}{d t}=j(x)-l(x)
$$

where

$$
\begin{aligned}
& x=\left(H_{k}, G_{k}, D_{k}\right)^{T}, \\
& j(x)=\left(\begin{array}{c}
k \rho(t)\left(\left(1-H_{k}-G_{k}-D_{k}\right)\right. \\
0 \\
0
\end{array}\right), \\
& l(x)=\left(\begin{array}{c}
((1-\chi) \eta+\chi \varepsilon+\delta) H_{k}-\psi \varphi G_{k} \\
(\psi \mu+\gamma+\psi \varphi+\delta) G_{k}-(1-\chi) \eta H_{k} \\
(\lambda+\delta) D_{k}-\psi \mu G_{k}
\end{array}\right) .
\end{aligned}
$$

At $E_{0}$, the Jacobian matrices of $j(x)$ and $l(x)$ are got

$$
J=D j\left(E_{0}\right)=\left(\begin{array}{ccc}
J_{11} & J_{12} & 0 \\
0 & 0 & 0 \\
0 & 0 & 0
\end{array}\right),
$$




$$
L=D l\left(E_{0}\right)=\left(\begin{array}{ccc}
L_{11} & L_{12} & 0 \\
L_{21} & L_{22} & 0 \\
0 & L_{32} & L_{33}
\end{array}\right),
$$

where

$$
\begin{gathered}
J_{11}=\frac{\beta_{1}}{\langle k\rangle}\left(\begin{array}{cccc}
Q(1) & 2 Q(2) & \cdots & n Q(n) \\
2 Q(1) & 2^{2} Q(2) & \cdots & 2 n Q(n) \\
\vdots & \vdots & \ddots & \vdots \\
n Q(1) & 2 n Q(2) & \cdots & n^{2} Q(n)
\end{array}\right), \\
J_{12}=\frac{\beta_{2}}{\langle k\rangle}\left(\begin{array}{cccc}
Q(1) & 2 Q(2) & \cdots & n Q(n) \\
2 Q(1) & 2^{2} Q(2) & \cdots & 2 n Q(n) \\
\vdots & \vdots & \ddots & \vdots \\
n Q(1) & 2 n Q(2) & \cdots & n^{2} Q(n)
\end{array}\right) .
\end{gathered}
$$

Here,

$$
\begin{array}{lll}
L_{11}=((1-\chi) \eta+\psi \varepsilon+\delta) I, & L_{12}=-\psi \varphi I, & L_{21}=-((1-\chi) \eta) I, \\
L_{22}=(\psi \mu+\gamma+\psi \varphi+\delta) I, & L_{32}=-\psi \mu I, & L_{33}=(\lambda+\delta) I,
\end{array}
$$

where $I$ is an identity matrix. So, we can calculate the basic reproductive number denoted by

$$
R_{0}=\rho\left(J L^{-1}\right)=\frac{\left\langle k^{2}\right\rangle}{\langle k\rangle} \frac{(\psi \mu+\gamma+\psi \varphi+\delta) \beta_{1}+\eta(1-\chi) \beta_{2}}{(\psi \mu+\gamma+\delta)((1-\chi) \eta+\chi \varepsilon+\delta)+\psi \varphi(\chi \varepsilon+\delta)},
$$

where $\left\langle k^{2}\right\rangle=\sum_{k} k^{2} Q(k)$.

Next, it is clear that system (4) has a gambling-free equilibrium $E_{0}(1,0,0,0)$. To get the gambling-prevailing equilibrium $E_{+}\left(S_{k}^{*}, H_{k}^{*}, G_{k}^{*}, D_{k}^{*}\right)$, system (4) satisfies

$$
\frac{d S_{k}(t)}{d t}=0, \quad \frac{d H_{k}(t)}{d t}=0, \quad \frac{d G_{k}(t)}{d t}=0, \quad \frac{d D_{k}(t)}{d t}=0 .
$$

So, we can know

$$
\left\{\begin{array}{l}
\delta+\lambda D_{k}(t)+\gamma G_{k}(t)+\chi \varepsilon H_{k}(t)-(k \rho(t)+\delta) S_{k}(t)=0, \\
k \rho(t) S_{k}(t)+\psi \varphi G_{k}(t)-((1-\chi) \eta+\chi \varepsilon+\delta) H_{k}(t)=0, \\
(1-\chi) \eta H_{k}(t)-(\psi \mu+\gamma+\psi \varphi+\delta) G_{k}(t)=0, \\
\psi \mu G_{k}(t)-(\lambda+\delta) D_{k}(t)=0 .
\end{array}\right.
$$

According to the above equation, we get

$$
\left\{\begin{array}{l}
S_{k}(t)=\frac{((1-\chi) \eta+\psi \varepsilon+\delta)(\psi \mu+\gamma+\psi \varphi+\delta)-\psi \varphi \eta(1-\chi)}{k \rho(t)(1-\chi) \eta} G_{k}(t), \\
H_{k}(t)=\frac{\psi \mu+\gamma+\psi \varphi+\delta}{(1-\chi) \eta} G_{k}(t), \\
D_{k}(t)=\frac{\psi \mu}{\lambda+\delta} G_{k}(t) .
\end{array}\right.
$$


By using to the normalization condition $S_{k}^{*}(t)+H_{k}^{*}(t)+G_{k}^{*}(t)+D_{k}^{*}(t)=1$, it gets

$$
\left\{\begin{array}{l}
S_{k}^{*}(t)=\frac{((1-\chi) \eta+\chi \varepsilon+\delta)(\psi \mu+\gamma+\delta)(\lambda+\delta)+\psi \varphi(\lambda+\delta)(\chi \varepsilon+\delta)}{B_{k}} \\
H_{k}^{*}(t)=\frac{k \rho(t)(\lambda+\delta)(\psi \mu+\gamma+\psi \varphi+\delta)}{B_{k}} \\
G_{k}^{*}(t)=\frac{k \rho(t) \eta(1-\chi)(\lambda+\delta)}{B_{k}} \\
D_{k}^{*}(t)=\frac{k \rho(t) \psi \mu \eta(1-\chi)}{B_{k}}
\end{array}\right.
$$

And

$$
\begin{aligned}
B_{k}= & k \rho(t)((\lambda+\delta)(\psi \mu+\gamma+\psi \varphi+\delta)+\psi \mu \eta(1-\chi)+\eta(1-\chi)(\lambda+\delta)) \\
& +\psi \varphi(\lambda+\delta)(\chi \varepsilon+\delta)+((1-\chi) \eta+\chi \varepsilon+\delta)(\psi \mu+\gamma+\delta)(\lambda+\delta)
\end{aligned}
$$

where $\rho(t)=\sum_{k} k Q(k)\left(\beta_{1} \theta_{1}+\beta_{2} \theta_{2}\right) /\langle k\rangle$. By substituting the second equation of system (16) into Eq. (2), we get

$$
\theta_{1}^{*}(t)=\frac{1}{\langle k\rangle} \sum_{k} k^{2} Q(k) \cdot \frac{\rho(t)(\lambda+\delta)(\psi \mu+\gamma+\psi \varphi+\delta)}{B_{k}} .
$$

According to $\theta_{1}(t)=\frac{\sum_{k} k Q(k) H_{k}(t)}{\sum_{k} s Q(s)}=\frac{1}{\langle k\rangle} \sum_{k} k Q(k) H_{k}(t)$ and $\theta_{2}(t)=\frac{\sum_{k} k Q(k) G_{k}(t)}{\sum_{k} s Q(s)}=$ $\frac{1}{\langle k\rangle} \sum_{k} k Q(k) G_{k}(t)$, we can get $\theta_{2}^{*}=\frac{\eta(1-\chi)}{\psi \mu+\gamma+\psi \varphi+\chi} \theta_{1}^{*}$. Then, let $\theta_{1}^{*} \triangleq f\left(\theta_{1}^{*}\right)$, obviously, $\theta_{1}^{*}=0$ is a solution. In order for $\theta_{1}^{*} \triangleq f\left(\theta_{1}^{*}\right)$ to have a nontrivial solution, the following conditions should be satisfied:

$$
\left.\frac{d f\left(\theta_{1}^{*}\right)}{d \theta_{1}^{*}}\right|_{\theta_{1}^{*}=0}>1 \text { and } f(1) \leq 1
$$

So, we get

$$
R_{0}=\frac{\left\langle k^{2}\right\rangle}{\langle k\rangle} \frac{(\psi \mu+\gamma+\psi \varphi+\delta) \beta_{1}+\eta(1-\chi) \beta_{2}}{(\psi \mu+\gamma+\delta)((1-\chi) \eta+\chi \varepsilon+\delta)+\psi \varphi(\chi \varepsilon+\delta)}>1
$$

According to Eq. (16), we know $0<S_{k}^{*}, H_{k}^{*}, G_{k}^{*}, D_{k}^{*}<1$. System (4) has the gamblingprevailing equilibrium $E_{+}\left(S_{k}^{*}, H_{k}^{*}, G_{k}^{*}, D_{k}^{*}\right)$. Then, when the basic regeneration number $R_{0}>$ 1 , there is a unique positive equilibrium $E_{+}\left(S_{k}^{*}, H_{k}^{*}, G_{k}^{*}, D_{k}^{*}\right)$. The proof is completed.

Theorem 2 When $R_{0}<1$, the gambling-free equilibrium $E_{0}$ is global asymptotically stable. When $R_{0}>1$, online gambling phenomenon is persistent, which means there is a constant $\phi>0, \liminf _{t \rightarrow \infty} \sum_{k}(H(t)+G(t)) \geq \phi$.

Proof For simplicity, let $Q_{i}=i Q(i) /\langle k\rangle$. For the gambling-free equilibrium, system (7) has the Jacobian matrix of $3 n \times 3 n$ as follows:

$$
G=\left(\begin{array}{ccc}
B_{11} & \cdots & B_{1 n} \\
\vdots & \ddots & \vdots \\
B_{n 1} & \cdots & B_{n n}
\end{array}\right)
$$


where

$$
\begin{aligned}
& B_{11}=\left(\begin{array}{ccc}
-((1-\chi) \eta+\chi \varepsilon+\delta)+\beta_{1} Q_{1} & \psi \varphi+\beta_{2} Q_{1} & 0 \\
(1-\chi) \eta & -(\psi \mu+\gamma+\psi \varphi+\delta) & 0 \\
0 & \psi \mu & -(\lambda+\delta)
\end{array}\right), \\
& B_{1 n}=\left(\begin{array}{ccc}
\beta_{1} Q_{n} & \beta_{2} Q_{n} & 0 \\
0 & 0 & 0 \\
0 & 0 & 0
\end{array}\right), \\
& B_{n 1}=\left(\begin{array}{ccc}
n \beta_{1} Q_{1} & n \beta_{2} Q_{1} & 0 \\
0 & 0 & 0 \\
0 & 0 & 0
\end{array}\right), \\
& B_{n n}=\left(\begin{array}{ccc}
-((1-\chi) \eta+\chi \varepsilon+\delta)+n \beta_{1} Q_{n} & \psi \varphi+n \beta_{2} Q_{n} & 0 \\
(1-\chi) \eta & -(\psi \mu+\gamma+\psi \varphi+\delta) & 0 \\
0 & \psi \mu & -(\lambda+\delta)
\end{array}\right) .
\end{aligned}
$$

So, the characteristic polynomial of the gambling-free equilibrium $E_{0}$ is

$$
(z+\lambda+\delta)^{n-1}(z+\psi \mu+\gamma+\psi \varphi+\delta)^{n-1}(z+(1-\chi) \eta+\chi \varepsilon+\delta)^{n-1}\left(z^{3}+s z^{2}+p z+q\right)=0,
$$

where $s=((1-\chi) \eta+\chi \varepsilon+\delta)+(\psi \mu+\gamma+\psi \varphi+\delta)+(\lambda+\delta)-\beta_{1} \sum_{i=1}^{n} i Q_{i}$, and

$$
\begin{aligned}
p= & ((1-\chi) \eta+\chi \varepsilon+\lambda+2 \delta)(\psi \mu+\gamma+\psi \varphi+\delta)+((1-\chi) \eta+\chi \varepsilon+\delta)(\lambda+\delta) \\
& -\psi \varphi \eta(1-\chi)-\left((\psi \mu+\gamma+\psi \varphi+\lambda+2 \delta) \beta_{1}+\eta(1-\chi) \beta_{2}\right) \sum_{i=1}^{n} i Q_{i}, \\
q= & ((1-\chi) \eta+\chi \varepsilon+\delta)(\psi \mu+\gamma+\psi \varphi+\delta)(\lambda+\delta)-\psi \varphi \eta(1-\chi)(\lambda+\delta) \\
& -\left((\psi \mu+\gamma+\psi \varphi+\delta) \beta_{1}+\eta(1-\chi) \beta_{2}\right)(\lambda+\delta) \sum_{i=1}^{n} i Q_{i} .
\end{aligned}
$$

Obviously, when $R_{0}<1, q>0$. It also means

$$
((1-\chi) \eta+\chi \varepsilon+\delta)+(\psi \mu+\gamma+\psi \varphi+\delta)+(\lambda+\delta)>\beta_{1} \sum_{i=1}^{n} i Q_{i}
$$

and

$$
\begin{gathered}
((1-\chi) \eta+\chi \varepsilon+\lambda+2 \delta)(\psi \mu+\gamma+\psi \varphi+\delta)+((1-\chi) \eta+\chi \varepsilon+\delta)(\lambda+\delta) \\
>\psi \varphi \eta(1-\chi)+\left((\psi \mu+\gamma+\psi \varphi+\lambda+2 \delta) \beta_{1}+\eta(1-\chi) \beta_{2}\right) \sum_{i=1}^{n} i Q_{i} .
\end{gathered}
$$

In other words, we get $s>0, q>0$, and $p>0$. According to the above proof, the real eigenvalues $\lambda$ of matrix $B$ are all negative when $R_{0}<1$. Furthermore, there is a unique positive eigenvalue $\lambda$ of matrix $B$ if $R_{0}>1$. By using the Perron-Frobenius theorem, the maximal real part of all eigenvalues of $\lambda$ is positive only if $R_{0}>1$. Through the theorem of Lajmanovich and York [25], we can get the results. The proof is completed. 
Theorem 3 ([26]) Suppose that $\left(H_{k}(t), G_{k}(t), D_{k}(t)\right)$ is the solution of system (7), which satisfies Eq. (5) with $H_{k}(0)>0$ or $G_{k}(0)>0$. If $R_{0}>1$, then $\lim _{t \rightarrow \infty}\left(H_{k}(t), G_{k}(t), D_{k}(t)\right)=$ $\left(H_{k}^{*}, G_{k}^{*}, D_{k}^{*}\right)$, where $\left(H_{k}^{*}, G_{k}^{*}, D_{k}^{*}\right)$ is the gambling-prevailing equilibrium of system (7) for $k=1,2, \ldots, n$.

Proof In the proof, let us assume that $k$ is integer between 1 and $n$. According to Theorem 2 , a positive constant $0<\alpha<1 / 3$ and a sufficiently large constant $T>0$ exist to satisfy $H_{k}(t) \geq \alpha$ and $G_{k}(t) \geq \alpha$ for $t>T$. Thus, $\rho(t)>\alpha\left(\beta_{1}+\beta_{2}\right)$ for $t>T$. Submitting this into the first equation of system (7), it is easy to get

$$
\frac{d H_{k}(t)}{d t} \leq k\left(\beta_{1}+\beta_{2}\right)\left(1-H_{k}(t)\right)-((1-\chi) \eta+\chi \varepsilon+\delta) H_{k}(t)
$$

for $t>T$.

According to the standard comparison theorem in the theory of differential equations, for any given positive constant

$$
0<\alpha_{1}<\frac{(1-\chi) \eta+\chi \varepsilon+\delta}{2\left[k\left(\beta_{1}+\beta_{2}\right)+((1-\chi) \eta+\chi \varepsilon+\delta)\right]},
$$

there exists $t_{1}>T$, so $H_{k}(t) \leq M_{k}^{(1)}-\alpha_{1}$ for $t>t_{1}$, where

$$
M_{k}^{(1)}=\frac{k\left(\beta_{1}+\beta_{2}\right)}{k\left(\beta_{1}+\beta_{2}\right)+((1-\chi) \eta+\chi \varepsilon+\delta)}+2 \alpha_{1}<1 .
$$

From system (7), it is easy to obtain

$$
\frac{d G_{k}(t)}{d t} \leq(1-\chi) \eta\left(1-G_{k}(t)\right)-(\psi \mu+\gamma+\psi \varphi+\delta) G_{k}(t)
$$

for $t>t_{1}$.

So, the constant

$$
0<\alpha_{2}<\min \left\{1 / 2, \alpha_{1}, \frac{\psi \mu+\gamma+\psi \varphi+\delta}{2((1-\chi) \eta+(\psi \mu+\gamma+\psi \varphi+\delta))}\right\},
$$

there exists $t_{2}>t_{1}$, so $G_{k}(t) \leq A_{k}^{(1)}-\alpha_{2}$ for $t>t_{2}$, where

$$
A_{k}^{(1)}=\frac{\eta(1-\chi)}{\eta(1-\chi)+(\psi \mu+\gamma+\psi \varphi+\delta)}+2 \alpha_{2}<1 \text {. }
$$

From system (7), it is easy to obtain

$$
\frac{d D_{k}(t)}{d t} \leq \psi \mu\left(1-D_{k}(t)\right)-(\lambda+\delta) D_{k}(t)
$$

for $t>t_{2}$.

Consequently, for constant

$$
0<\alpha_{3}<\min \left\{1 / 3, \alpha_{2}, \frac{\lambda+\delta}{2(\psi \mu+(\lambda+\delta))}\right\}
$$


there exists $t_{3}>t_{2}$ such that $D_{k}(t) \leq V_{k}^{(1)}-\alpha_{3}$ for $t>t_{3}$, where

$$
V_{k}^{(1)}=\frac{\psi \mu}{\psi \mu+(\lambda+\delta)}+2 \alpha_{3}<1
$$

Then, replacing $H_{k}(t) \geq \alpha, G_{k}(t) \geq \alpha$ and $\rho(t)>\alpha\left(\beta_{1}+\beta_{2}\right)$ into the first equation of system (7), we get

$$
\begin{aligned}
\frac{d H_{k}(t)}{d t} \geq & k \alpha\left(\beta_{1}+\beta_{2}\right)\left(1-H_{k}(t)-G_{k}(t)-D_{k}(t)\right) \\
& +\psi \varphi G_{k}(t)-((1-\chi) \eta+\chi \varepsilon+\delta) H_{k}(t) \\
\geq & k \alpha\left(\beta_{1}+\beta_{2}\right)\left(1-A_{k}^{(1)}-V_{k}^{(1)}\right)+\psi \varphi A_{k}^{(1)} \\
& -\left(k \alpha\left(\beta_{1}+\beta_{2}\right)+(1-\chi) \eta+\chi \varepsilon+\delta\right) H_{k}(t)
\end{aligned}
$$

for $t>T$.

Therefore, for constant

$$
0<\alpha_{4}<\min \left\{1 / 4, \alpha_{3}, \frac{k \alpha\left(\beta_{1}+\beta_{2}\right)\left(1-A_{k}^{(1)}-V_{k}^{(1)}\right)+\psi \varphi A_{k}^{(1)}}{2\left[k \alpha\left(\beta_{1}+\beta_{2}\right)+(1-\chi) \eta+\chi \varepsilon+\delta\right]}\right\},
$$

there exists $t_{4}>t_{3}$ such that $H_{k}(t) \geq m_{k}^{(1)}+\alpha_{4}$ for $t>t_{4}$, where

$$
m_{k}^{(1)}=\frac{k \alpha\left(\beta_{1}+\beta_{2}\right)\left(1-N_{k}^{(1)}-V_{k}^{(1)}\right)+\psi \varphi N_{k}^{(1)}}{k \alpha\left(\beta_{1}+\beta_{2}\right)+(1-\chi) \eta+\chi \varepsilon+\delta}-2 \alpha_{4}>0 .
$$

Therefore

$$
\frac{d G_{k}(t)}{d t} \geq \eta(1-\chi) m_{k}^{(1)}-(\psi \mu+\gamma+\psi \varphi+\delta) G_{k}(t)
$$

for $t>t_{4}$.

Hence, for constant

$$
0<\alpha_{5}<\min \left\{1 / 5, \alpha_{4}, \frac{\eta(1-\chi) m_{k}^{(1)}}{2(\psi \mu+\gamma+\psi \varphi+\delta)}\right\},
$$

there exists $t_{5}>t_{4}$ such that $G_{k}(t) \geq a_{k}^{(1)}+\alpha_{5}$ for $t>t_{5}$, where

$$
a_{k}^{(1)}=\frac{\eta(1-\chi) x_{k}^{(1)}}{\psi \mu+\gamma+\psi \varphi+\delta}-2 \alpha_{5}>0 .
$$

Similarly,

$$
\frac{d D_{k}(t)}{d t} \geq \psi \mu a_{k}^{(1)}-(\lambda+\delta) D_{k}(t)
$$

for $t>t_{5}$.

Consequently, for constant

$$
0<\alpha_{6}<\min \left\{1 / 6, \alpha_{5}, \frac{\psi \mu a_{k}^{(1)}}{2(\lambda+\delta)}\right\},
$$


there exists $t_{6}>t_{5}$ such that $D_{k}(t) \geq v_{k}^{(1)}+\alpha_{6}$ for $t>t_{6}$, where

$$
v_{k}^{(1)}=\frac{\psi \mu a_{k}^{(1)}}{\lambda+\delta}-2 \alpha_{6}>0 .
$$

Because $\alpha$ is a small constant, we can get $0<m_{k}^{(1)}<M_{k}^{(1)}<1,0<a_{k}^{(1)}<A_{k}^{(1)}<1$, and $0<v_{k}^{(1)}<V_{k}^{(1)}<1$.

Let

$$
u^{(j)}=\sum_{i=1}^{n} Q_{i}\left(\beta_{1} m_{i}^{(j)}+\beta_{2} a_{i}^{(j)}\right), \quad U^{(j)}=\sum_{i=1}^{n} Q_{i}\left(\beta_{1} M_{i}^{(j)}+\beta_{2} A_{i}^{(j)}\right), \quad j=1,2, \ldots
$$

From the above discussion, we have

$$
0<u^{(1)} \leq \rho(t) \leq U^{(1)}<\beta_{1}+\beta_{2}
$$

and $t>t_{6}$.

And, according to system (7), we can get

$$
\frac{d H_{k}(t)}{d t} \leq k U^{(1)}\left(1-a_{k}^{(1)}-v_{k}^{(1)}\right)+\psi \varphi a_{k}^{(1)}-\left(k U^{(1)}+(1-\chi) \eta+\chi \varepsilon+\delta\right) H_{k}(t)
$$

for $t>t_{6}$.

Consequently, for constant $0<\alpha_{7}<\min \left\{1 / 7, \alpha_{6}\right\}$, there exists $t_{7}>t_{6}$ such that

$$
H_{k}(t) \leq M_{k}^{(2)} \triangleq \min \left\{M_{k}^{(1)}-\alpha_{1}, \frac{k U^{(1)}\left(1-a_{k}^{(1)}-v_{k}^{(1)}\right)+\psi \varphi y_{k}^{(1)}}{k U^{(1)}+(1-\chi) \eta+\chi \varepsilon+\delta}+\alpha_{7}\right\}
$$

for $t>t_{7}$.

Thus,

$$
\frac{d G_{k}(t)}{d t} \leq \eta(1-\chi) M_{k}^{(2)}-(\psi \mu+\gamma+\psi \varphi+\delta) G_{k}(t)
$$

for $t>t_{7}$.

Consequently, for constant $0<\alpha_{8}<\min \left\{1 / 8, \alpha_{7}\right\}$, there exists $t_{8}>t_{7}$ such that

$$
G_{k}(t) \leq A_{k}^{(2)} \triangleq \min \left\{A_{k}^{(1)}-\alpha_{2}, \frac{\eta(1-\chi) M_{k}^{(2)}}{\psi \mu+\gamma+\psi \varphi+\delta}+\alpha_{8}\right\}
$$

for $t>t_{8}$.

As a result, it follows that

$$
\frac{d D_{k}(t)}{d t} \leq \psi \mu A_{k}^{(2)}-(\lambda+\delta) D_{k}(t)
$$

for $t>t_{8}$.

Therefore, for constant $0<\alpha_{9}<\min \left\{1 / 9, \alpha_{8}\right\}$, there exists $t_{9}>t_{8}$ such that

$$
D_{k}(t) \leq V_{k}^{(2)} \triangleq \min \left\{V_{k}^{(1)}-\alpha_{3}, \frac{\psi \mu A_{k}^{(2)}}{\lambda+\delta}+\alpha_{9}\right\}
$$

for $t>t_{9}$. 
According to system (7), we can get

$$
\frac{d H_{k}(t)}{d t} \geq k u^{(1)}\left(1-A_{k}^{(2)}-V_{k}^{(2)}\right)+\psi \varphi A_{k}^{(2)}-\left(k u^{(1)}+(1-\chi) \eta+\chi \varepsilon+\delta\right) H_{k}(t)
$$

for $t>t_{9}$.

Hence, for constant

$$
0<\alpha_{10}<\min \left\{1 / 10, \alpha_{9}, \frac{k u^{(1)}\left(1-A_{k}^{(2)}-V_{k}^{(2)}\right)+\psi \varphi A_{k}^{(2)}}{2\left(k u^{(1)}+(1-\chi) \eta+\chi \varepsilon+\delta\right)}\right\},
$$

there exists $t_{10}>t_{9}$, and $H_{k}(t) \geq m_{k}^{(2)}+\alpha_{10}, t>t_{10}$, where

$$
m_{k}^{(2)}=\max \left\{m_{k}^{(1)}+\alpha_{4}, \frac{k u^{(1)}\left(1-A_{k}^{(2)}-V_{k}^{(2)}\right)+\psi \varphi A_{k}^{(2)}}{k u^{(1)}+(1-\chi) \eta+\chi \varepsilon+\delta}-2 \alpha_{10}\right\} .
$$

Thus,

$$
\frac{d G_{k}(t)}{d t} \geq(1-\chi) \eta m_{k}^{(2)}-(\psi \mu+\gamma+\psi \varphi+\delta) G_{k}(t)
$$

for $t>t_{10}$

So, for constant

$$
0<\alpha_{11}<\min \left\{1 / 11, \alpha_{10}, \frac{(1-\chi) \eta m_{k}^{(2)}}{2(\psi \mu+\gamma+\psi \varphi+\delta)}\right\}
$$

there exists $t_{11}>t_{10}$, and $G_{k}(t) \geq a_{k}^{(2)}+\alpha_{11}, t>t_{11}$, where

$$
a_{k}^{(2)}=\max \left\{a_{k}^{(1)}+\alpha_{5}, \frac{(1-\chi) \eta m_{k}^{(2)}}{\psi \mu+\gamma+\psi \varphi+\delta}-2 \alpha_{11}\right\} .
$$

Similarly,

$$
\frac{d D_{k}(t)}{d t} \geq \psi \mu a_{k}^{(2)}-(\lambda+\delta) D_{k}(t)
$$

for $t>t_{11}$.

Therefore, for constant

$$
0<\alpha_{12}<\min \left\{1 / 12, \alpha_{11}, \frac{\psi \mu a_{k}^{(2)}}{2(\lambda+\delta)}\right\}
$$

there exists $t_{12}>t_{11}$, and $D_{k}(t) \geq v_{k}^{(2)}+\alpha_{12}, t>t_{12}$, where

$$
v_{k}^{(2)}=\max \left\{v_{k}^{(1)}+\alpha_{6}, \frac{\psi \mu a_{k}^{(2)}}{\lambda+\delta}-2 \alpha_{12}\right\} .
$$

According to the above discussion and analyses, we can obtain six sequences: $\left\{M_{k}^{(r)}\right\}$, $\left\{A_{k}^{(r)}\right\},\left\{V_{k}^{(r)}\right\},\left\{m_{k}^{(r)}\right\},\left\{a_{k}^{(r)}\right\}$, and $\left\{v_{k}^{(r)}\right\}$. We can find that the first three sequences are monotone increasing and the last three sequences are strictly monotone decreasing, and there 
is a sufficiently large positive integer $L$ such that, for $r \geq L$ :

$$
\left\{\begin{array}{l}
M_{k}^{(r)}=\frac{k U^{(r-1)}\left(1-a_{k}^{(r-1)}-v_{k}^{(r-1)}\right)+\psi \varphi a_{k}^{(r-1)}}{k U^{(r-1)}+(1-\chi) \eta+\chi \varepsilon+\delta}+\alpha_{6 r-5}, \\
A_{k}^{(r)}=\frac{(1-\chi) \eta M_{k}^{(r)}}{\psi \mu+\gamma+\psi \varphi+\delta}+\alpha_{6 r-4}, \\
V_{k}^{(r)}=\frac{\psi \mu A_{k}^{(r)}}{\lambda+\delta}+\alpha_{6 r-3}, \\
m_{k}^{(r)}=\frac{k u^{(r-1)}\left(1-A_{k}^{(r)}-V_{k}^{(r)}\right)+\psi \varphi A_{k}^{(r)}}{k u^{(r-1)}+(1-\chi) \eta+\chi \varepsilon+\delta}-2 \alpha_{6 r-2}, \\
a_{k}^{(r)}=\frac{(1-\chi) \eta m_{k}^{(r)}}{\psi \mu+\gamma+\psi \varphi+\delta}-2 \alpha_{6 r-1}, \\
v_{k}^{(r)}=\frac{\psi \mu a_{k}^{(r)}}{\lambda+\delta}-2 \alpha_{6 r} .
\end{array}\right.
$$

It is easy to find that

$$
\left\{\begin{array}{l}
m_{k}^{(r)} \leq H_{k}(t) \leq M_{k}^{(r)}, \\
a_{k}^{(r)} \leq G_{k}(t) \leq A_{k}^{(r)}, \quad \text { where } t>t_{6 r} . \\
v_{k}^{(r)} \leq D_{k}(t) \leq V_{k}^{(r)},
\end{array}\right.
$$

Since the sequential limits of system (62), thus let $\lim _{t \rightarrow \infty} \Omega_{k}^{(r)}=\Omega_{k}$, where $\Omega_{k} \in\left\{M_{k}, A_{k}\right.$, $\left.V_{k}, m_{k}, a_{k}, v_{k}, U_{k}, u_{k}\right\}$ and $\Omega_{k}^{(r)} \in\left\{M_{k}^{(r)}, A_{k}^{(r)}, V_{k}^{(r)}, m_{k}^{(r)}, a_{k}^{(r)}, v_{k}^{(r)}, U_{k}^{(r)}, u_{k}^{(r)}\right\}$. Since $0<\alpha_{r}<1 / r$, it has $\alpha_{r} \rightarrow 0$ as $r \rightarrow \infty$. Supposing $r \rightarrow \infty$, it follows from (62) that

$$
\left\{\begin{array}{lll}
M_{k}=\frac{k U\left(1-a_{k}-v_{k}\right)+\psi \varphi a_{k}}{k U+(1-\chi) \eta+\chi \varepsilon+\delta}, & A_{k}=\frac{(1-\chi) \eta M_{k}}{\psi \mu+\gamma+\psi \varphi+\delta}, & V_{k}=\frac{\psi \mu A_{k}}{\lambda+\delta} \\
m_{k}=\frac{k u\left(1-A_{k}-V_{k}\right)+\psi \varphi A_{k}}{k u+(1-\chi) \eta+\chi \varepsilon+\delta}, & a_{k}=\frac{(1-\chi) \eta m_{k}}{\psi \mu+\gamma+\psi \varphi+\delta}, & v_{k}=\frac{\psi \mu a_{k}}{\lambda+\delta}
\end{array}\right.
$$

where

$$
u=\sum_{i=1}^{n} Q_{i}\left(\beta_{1} m_{i}+\beta_{2} a_{i}\right), \quad U=\sum_{i=1}^{n} Q_{i}\left(\beta_{1} M_{i}+\beta_{2} A_{i}\right) .
$$

What is more,

$$
\left\{\begin{aligned}
M_{k}= & \frac{1}{G_{k}}\left[k U(\lambda+\delta)^{2}(\psi \mu+\gamma+\psi \varphi+\delta)^{2}(k u+(1-\chi) \eta+\chi \varepsilon+\delta)\right. \\
& +k u(1-\chi)(\lambda+\delta)(\psi \mu+\gamma+\psi \varphi+\delta)(\psi \varphi \eta(\lambda+\delta)-k U(\eta(\lambda+\delta)+\psi \mu \eta))], \\
A_{k}= & \frac{1}{G_{k}}\left[k U \eta(1-\chi)(\psi \mu+\gamma+\psi \varphi+\delta)(\lambda+\delta)^{2}(k u+(1-\chi) \eta+\chi \varepsilon+\delta)\right. \\
& \left.+k u \eta(\lambda+\delta)(1-\chi)^{2}(\psi \varphi \eta(\lambda+\delta)-k U(\eta(\lambda+\delta)+\psi \mu \eta))\right], \\
m_{k}= & \frac{1}{G_{k}}\left[k u(\lambda+\delta)^{2}(\psi \mu+\gamma+\psi \varphi+\delta)^{2}(k U+(1-\chi) \eta+\chi \varepsilon+\delta)\right. \\
& +k U(1-\chi)(\lambda+\delta)(\psi \mu+\gamma+\psi \varphi+\delta)(\psi \varphi \eta(\lambda+\delta)-k u(\eta(\lambda+\delta)+\psi \mu \eta))], \\
a_{k}= & \frac{1}{G_{k}}\left[k u \eta(1-\chi)(\psi \mu+\gamma+\psi \varphi+\delta)(\lambda+\delta)^{2}(k U+(1-\chi) \eta+\chi \varepsilon+\delta)\right. \\
& \left.+k U \eta(\lambda+\delta)(1-\chi)^{2}(\psi \varphi \eta(\lambda+\delta)-k u(\eta(\lambda+\delta)+\psi \mu \eta))\right],
\end{aligned}\right.
$$

where

$$
\begin{aligned}
G_{k}= & (\lambda+\delta)^{2}(\psi \mu+\gamma+\psi \varphi+\delta)^{2}(k u+(1-\chi) \eta+\chi \varepsilon+\delta)(k U+(1-\chi) \eta+\chi \varepsilon+\delta) \\
& -(1-\chi)^{2}(\psi \varphi \eta(\lambda+\delta)-k U(\eta(\lambda+\delta)+\psi \mu \eta)) \\
& \times(\psi \varphi \eta(\lambda+\delta)-k u(\eta(\lambda+\delta)+\psi \mu \eta)) .
\end{aligned}
$$


From the above equation, we get $U=u$. So,

$$
\sum_{i=1}^{n} Q_{i}\left[\beta_{1}\left(M_{i}-m_{i}\right)+\beta_{2}\left(A_{i}-a_{i}\right)\right]=0
$$

which is equivalent to $M_{i}=m_{i}$ and $A_{i}=a_{i}$ for $1 \leq i \leq n$. Then, from systems (63) and (64), it can be concluded that

$$
\lim _{t \rightarrow \infty} H_{k}(t)=M_{k}=m_{k}, \quad \lim _{t \rightarrow \infty} G_{k}(t)=A_{k}=a_{k}, \quad \lim _{t \rightarrow \infty} D_{k}(t)=V_{k}=v_{k} .
$$

Finally, $U=u$ is substituted into system (65). For system (64), we can get $M_{k}=H_{k}^{*}, A_{k}=G_{k}^{*}$, and $V_{k}=D_{k}^{*}$. The proof is completed.

\section{Simulation results and analyses}

In this section, the analysis results are illustrated through numerical simulations. Based on a scale-free network, we have $Q(k)=\omega k^{-3}$ in system (1), and the parameter $\omega$ satisfies $\sum_{k=1}^{n} \omega k^{-3}=1, n=1000$.

In Fig. 2, we choose $\delta=0.2, \beta_{1}=0.1, \beta_{2}=0.1, \varepsilon=0.4, \chi=0.3, \eta=0.5, \varphi=0.1, \psi=0.7$, $\mu=0.6, \gamma=0.3, \lambda=0.1$ and obtain the basic reproductive number $R_{0}=0.9544<1$. Figure 2 shows that when $R_{0}<1, H_{150}$ and $G_{150}$ will equal to zero eventually, which means that the spread of online gambling phenomenon will eventually disappear.

In Fig. 3, we choose $\delta=0.1, \beta_{1}=0.2, \beta_{2}=0.5, \varepsilon=0.1, \chi=0.1, \eta=0.3, \varphi=0.2, \psi=0.4$, $\mu=0.6, \gamma=0.1, \lambda=0.1$ and obtain $R_{0}=6.1795>1$. The figure shows that when $R_{0}>1$, $H_{150}$ and $G_{150}$ will maintain positive recently, and the online gambling phenomenon will not disappear.

In Fig. 4(a) and (b), we choose $\delta=0.2, \beta_{1}=0.1, \beta_{2}=0.1, \varepsilon=0.4, \chi=0.3, \eta=0.5, \varphi=0.1$, $\psi=0.7, \mu=0.6, \gamma=0.3, \lambda=0.1$ and obtain $R_{0}=0.9544<1$. The figure shows trends of the hesitator $H(t)$ and the gambler $G(t)$ over time with different degree. And when $R_{0}<1$, online gambling phenomenon will ultimately disappear. In addition, the larger the degree is, the faster the spread of online gambling behavior.

In Fig. 5(a) and (b), we choose $\delta=0.1, \beta_{1}=0.2, \beta_{2}=0.5, \varepsilon=0.1, \chi=0.1, \eta=0.3, \varphi=0.2$, $\psi=0.4, \mu=0.6, \gamma=0.1, \lambda=0.1$ and obtain $R_{0}=6.1795>1$. The figure shows trends of the hesitator $H(t)$ and the gambler $G(t)$ over time with different degree. And when $R_{0}>1$,

Figure 2 The orbits of four states over time when $R_{0}=0.9544<1$

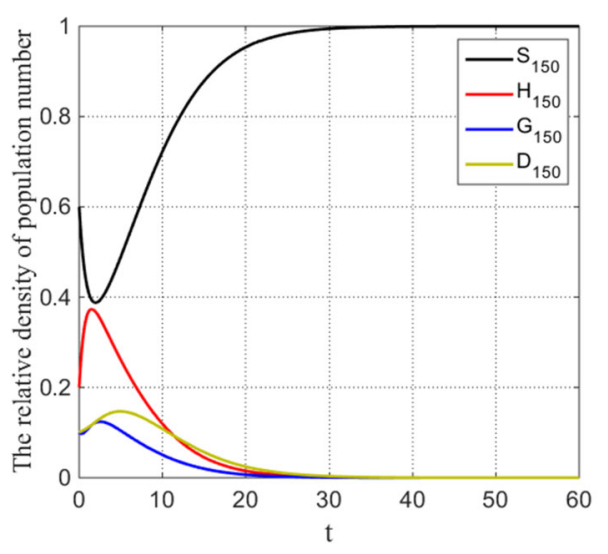


Figure 3 The orbits of four states over time when $R_{0}=6.1795>1$

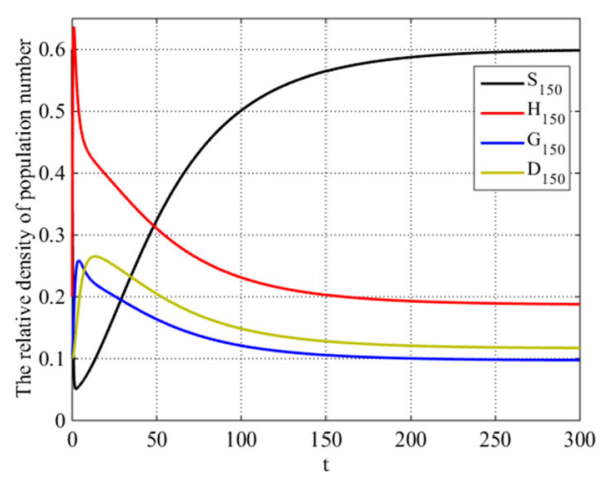

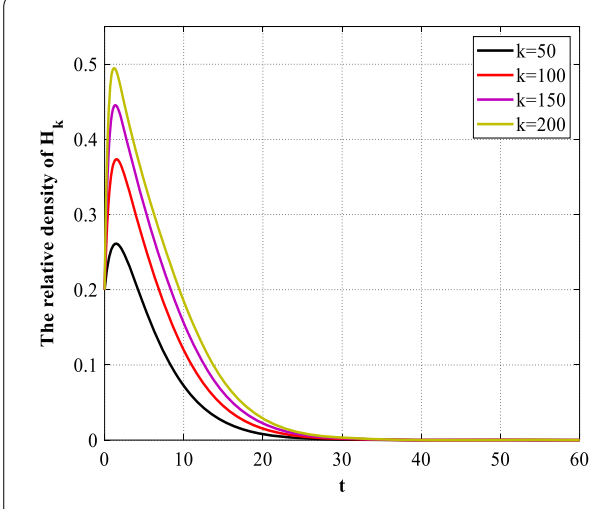

(a) Hesitators $H(t)$

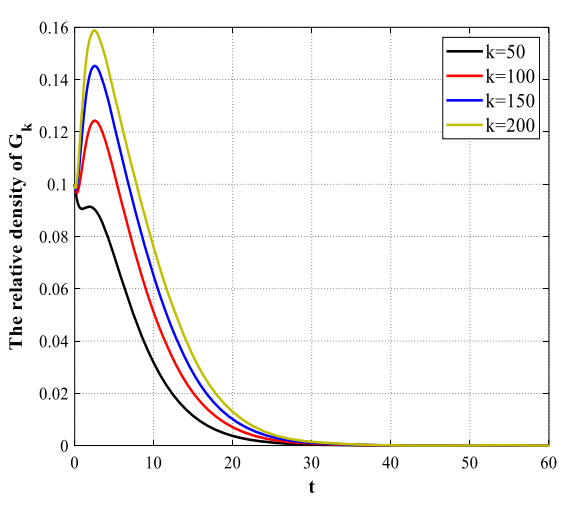

(b) Gamblers $G(t)$

Figure 4 The orbits of the hesitator or the gambler with different degree when $R_{0}<1$

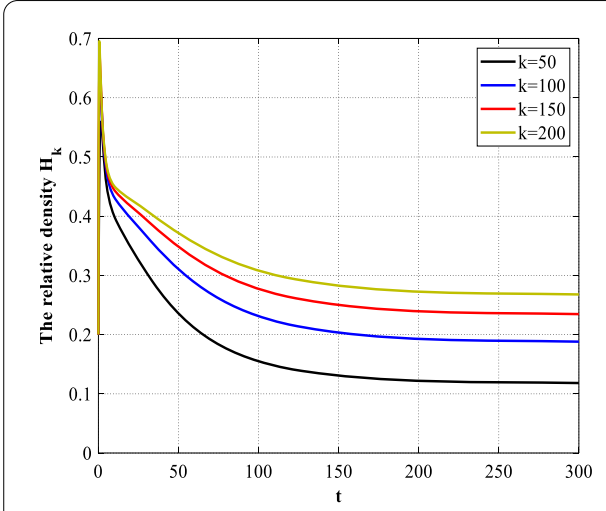

(a) Hesitators $H(t)$

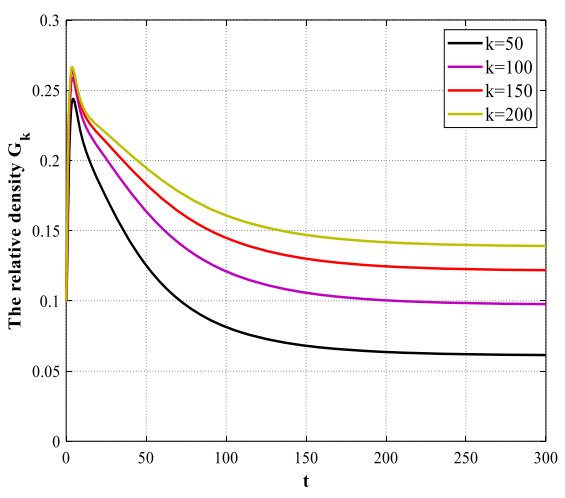

(b) Gamblers $G(t)$

Figure 5 The orbits of the hesitator or the gambler with different degree when $R_{0}>1$

online gambling phenomenon will be persistent. Moreover, more people are involved in gambling with the increasing of degree.

In Fig. 6(a) and (b), we choose $\delta=0.1, \beta_{1}=0.2, \beta_{2}=0.5, \varepsilon=0.1, \chi=0.1, \eta=0.3, \varphi=0.2$, $\mu=0.6, \gamma=0.1, \lambda=0.1$. The figure shows the change of the hesitator $H(t)$ and the gambler $G(t)$ with different probability $\psi$. With the growth of $\psi, H(t)$ will increase but $G(t)$ will fall to a constant. Apparently, larger $\psi$ can decrease the number of gamblers. 


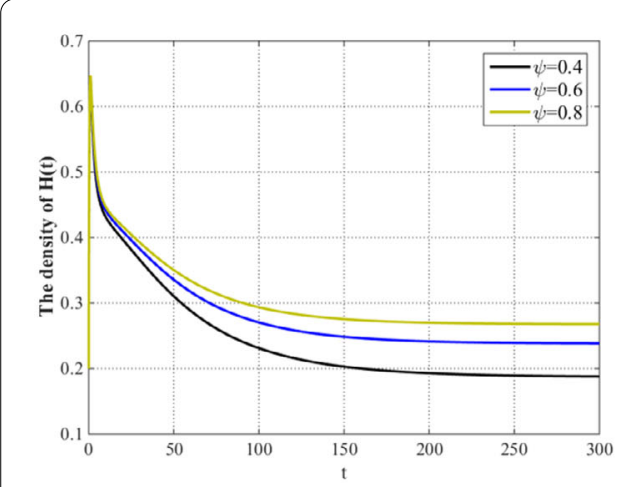

(a) Hesitators $H(t)$

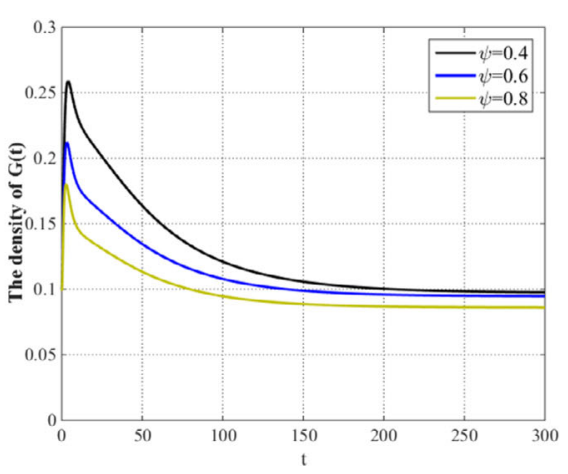

(b) Gamblers $G(t)$

Figure 6 Prevalence $H_{100}(t), G_{100}(t)$ versus $t$ corresponding to different $\psi$

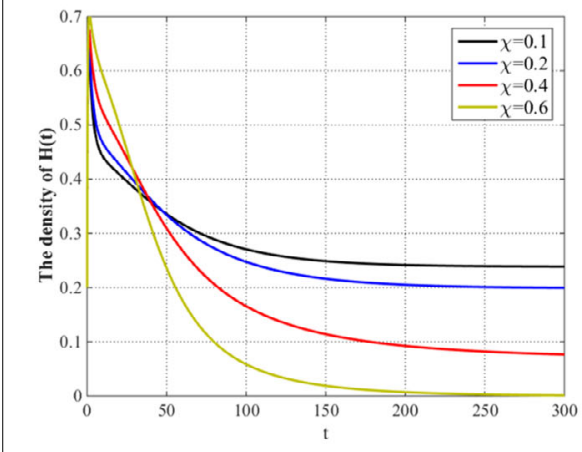

(a) Hesitators $H(t)$

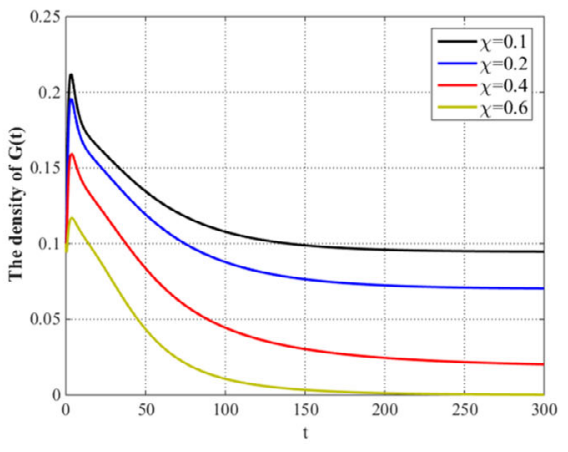

(b) Gamblers $G(t)$

Figure 7 Prevalence $H_{100}(t), G_{100}(t)$ versus $t$ corresponding to different $\chi$

In Fig. 7(a) and (b), we choose $\delta=0.1, \beta_{1}=0.2, \beta_{2}=0.5, \varepsilon=0.1, \eta=0.3, \varphi=0.2, \psi=0.6$, $\mu=0.6, \gamma=0.1, \lambda=0.1$. The figure shows the change of the hesitator $H(t)$ and the gambler $G(t)$ with different probability $\chi$. With the growth of $\chi, H(t)$ and $G(t)$ will fall to a constant. Apparently, larger $\chi$ can decrease the number of the hesitator and the gambler.

In Fig. 8, the parameters are chosen as $\delta=0.2, \varepsilon=0.4, \chi=0.3, \eta=0.5, \varphi=0.1, \psi=0.7$, $\mu=0.6, \gamma=0.3, \lambda=0.1$. We can see that larger $\beta_{1}$ or $\beta_{2}$ can lead to larger $R_{0}$, and $\beta_{1}$ has a greater impact on $R_{0}$. That is to say, the larger number of the hesitator $H(t)$ and the gambler $G(t)$ can speed up the spread of online gambling.

In Fig. 9(a) and (b), we choose $\delta=0.1, \beta_{1}=0.2, \beta_{2}=0.5, \varepsilon=0.1, \eta=0.3, \varphi=0.2, \mu=$ $0.6, \gamma=0.1, \lambda=0.1$. Apparently, larger $\chi$ or $\psi$ can lead to smaller $R_{0}, \chi$ has a greater impact on $R_{0}$. In other words, within a certain range of anti-gambling efforts, the antigambling policy helps to decrease the spread of online gambling, and the anti-gambling policy for the hesitator is more effective in reducing the spread of online gambling. It is more effective to decrease the spread of online gambling if they work together.

\section{Conclusion}

In this paper, we proposed a new $S H G D$ online gambling spreading model and analyzed the spreading dynamics of online gambling. We obtained the basic reproductive number $R_{0}$, gambling-free equilibrium $E_{0}$, and gambling-prevailing equilibrium $E_{+}$. If $R_{0}<1$, the 


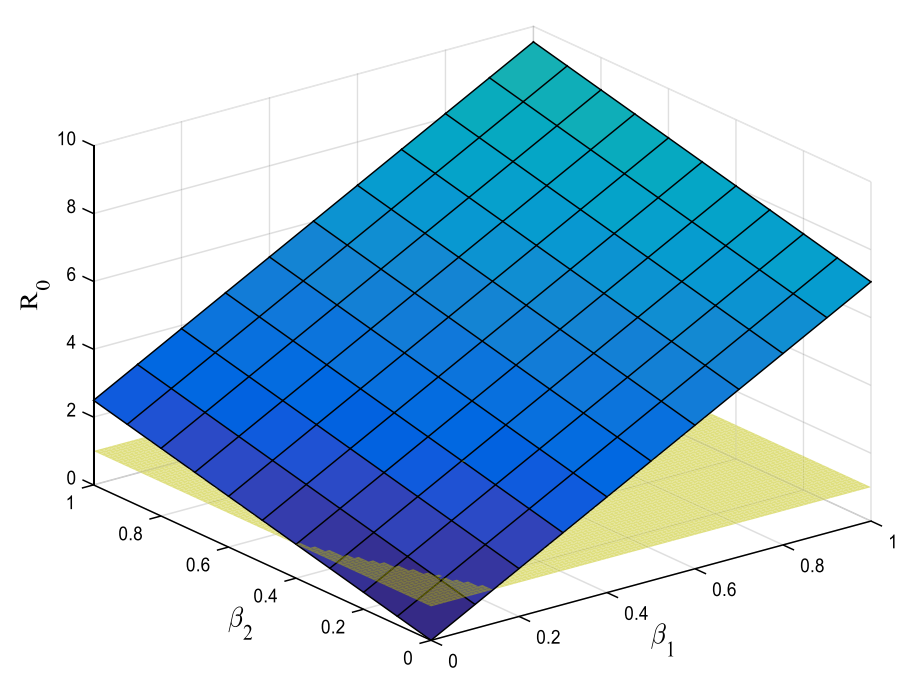

Figure 8 The relationship between the basic reproductive number $R_{0}$ and the parameters $\beta_{1}$ and $\beta_{2}$ on scale-free networks

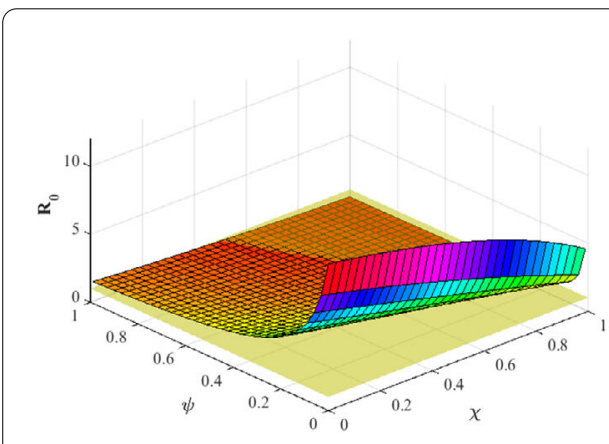

(a) Graphic model of $R_{0}$

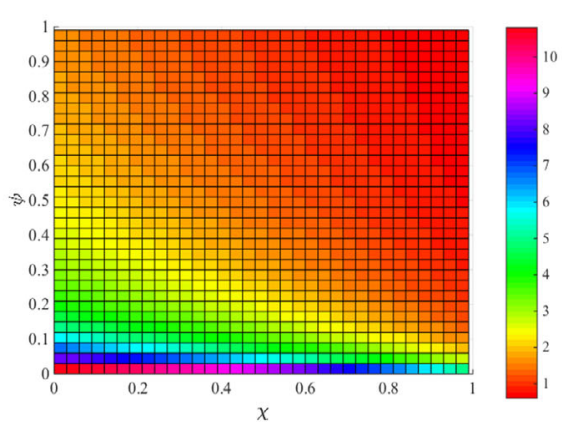

(b) Plane figure of $R_{0}$

Figure 9 The relationship between the basic reproductive number $R_{0}$ and the parameters $\psi$ and $\chi$ on scale-free networks

gambling-free equilibrium is globally asymptotically stable, i.e., online gambling spreading phenomenon will eventually disappear. If $R_{0}>1$, the spread of online gambling phenomenon is persistent and globally asymptotically stable, i.e., online gambling is a universal phenomenon. Smaller $\beta_{1}$ and $\beta_{2}$ can lead to the lower number of the disseminator, and $\beta_{1}$ has a greater impact than $\beta_{2}$. Furthermore, larger $\chi$ and $\psi$ can speed up the disappearance of online gambling phenomenon, especially $\chi$. That is, increasing the intensity of the anti-gambling policy on the hesitator or the gambler can restrain online gambling spreading, and the anti-gambling policy on the hesitator is more effective. This research results have important guiding significance in controlling the spreading of online gambling.

\section{Acknowledgements}

We thank the referees and the editor for their careful reading of the original manuscript and many valuable comments and suggestions that greatly improved the presentation of this paper.

\section{Funding}

This work is supported in part by the National Natural Science Foundation of China under grants 61672112 and 61873287. 
Availability of data and materials

Data sharing not applicable to this article as no datasets were generated or analyzed during the current study.

\section{Competing interests}

The authors declare that they have no competing interests.

\section{Authors' contributions}

YK performed the analysis and wrote the manuscript; TL designed the study; YW and XC developed the methodology; HW and YL helped perform the analysis with constructive discussions. All authors read and approved the final manuscript.

\section{Author details}

${ }^{1}$ School of Electronics and Information, Yangtze University, Jingzhou, 434023, P.R. China. ${ }^{2}$ College of Information Science and Engineering, Central South University, Changsha, 410083, P.R. China.

\section{Publisher's Note}

Springer Nature remains neutral with regard to jurisdictional claims in published maps and institutional affiliations.

Received: 3 June 2020 Accepted: 6 December 2020 Published online: 07 January 2021

\section{References}

1. Binde, P.: Exploring the impact of gambling advertising: an interview study of problem gamblers. Int. J. Mental Health Addict. 7(4), 541 (2009)

2. Young, M., Markham, F., Doran, B.: Too close to home? The relationships between residential distance to venue and gambling outcomes. Int. Gambl. Stud. 12(2), 257-273 (2012)

3. Griffiths, M.: Gambling technologies: prospects for problem gambling. J. Gambl. Stud. 15(3), 265-283 (1999)

4. Fiedler, I., Kairouz, S., Costes, M.J., Kristina, S.: Gambling spending and its concentration on problem gamblers. J. Bus. Res. 98, 82-91 (2019)

5. Hoffmann, J.P.: Religion and problem gambling in the US. Rev. Relig. Res. 41(4), 488-509 (2000)

6. Holtgraves, T.: Evaluating the problem gambling severity index. J. Gamb. Stud. 25(1), 105 (2009)

7. Rockloff, M.J., Schofield, G.: Factor analysis of barriers to treatment for problem gambling. J. Gamb. Stud. 20(2), $121-126$ (2004)

8. Hodgins, D.C., Currie, S.R., El-Guebaly, N.: Motivational enhancement and self-help treatments for problem gambling. J. Consult. Clin. Psychol. 69(1), 50 (2001)

9. Hodgins, D.C., El-Guebaly, N.: Natural and treatment-assisted recovery from gambling problems: a comparison of resolved and active gamblers. Addiction 95(5), 777-789 (2000)

10. King, S.A., Barak, A.: Compulsive Internet gambling: a new form of an old clinical pathology. CyberPsychol. Behav. 2(5), 441-456 (1999)

11. Dickson-Gillespie, L., Rugle, L., Rosenthal, R., Fong, T.: Preventing the incidence and harm of gambling problems. J. Primary Prevent. 29(1), 37-55 (2008)

12. Stehmann, J.: Identifying research streams in online gambling and gaming literature: a bibliometric analysis. Comput. Hum. Behav. 107, 106219 (2020)

13. Guan, Z.H., Sun, F.L., Wang, Y.W., Li, T.: Finite-time consensus for leader-following second-order multi-agent networks. IEEE Trans. Circuits Syst. I, Regul. Pap. 59(11), 2646-2654 (2012)

14. Pastor-Satorras, R., Castellano, C., Van, M.P., et al.: Epidemic processes in complex networks. Rev. Mod. Phys. 87(3), 925 (2015)

15. Zhan, X.S., Sun, X.X., Li, T., Wu, J., Jiang, X.W.: Optimal performance of networked control systems with bandwidth and coding constraints. ISA Trans. 59, 172-179 (2015)

16. Li, C: A study on time-delay rumor propagation model with saturated control function. Adv. Differ. Equ. 2017, 255 (2017)

17. Lin, T., Fan, C., Liu, C., et al.: Optimal control of a rumor propagation model with latent period in emergency event Adv. Differ. Equ. 2015, 54 (2015)

18. Wang, Y., Cao, J., Alsaedi, A., et al.: The spreading dynamics of sexually transmitted diseases with birth and death on heterogeneous networks. J. Stat. Mech. Theory Exp. 2017(2), 023502 (2017)

19. Liu, W., Li, T., Liu, X.: Spreading dynamics of a word-of-mouth model on scale-free networks. IEEE Access 6 , 65563-65572 (2018)

20. Wang, Y., Cao, J.: Global dynamics of a network epidemic model for waterborne diseases spread. Appl. Math. Comput. 237, 474-488 (2014)

21. King, D.L., Delfabbro, P.H.: Early exposure to digital simulated gambling: a review and conceptual model. Comput. Hum. Behav. 55, 198-206 (2016)

22. Lei, Y., Li, T., Wang, Y., Ye, G., Sun, S., Xia, Z.: Spreading dynamics of a CPFB group booking preferential information model on scale-free networks. IEEE Access 7, 156287-156300 (2019)

23. Liu, X., Li, T., Cheng, X., et al.: Spreading dynamics of a preferential information model with hesitation psychology on scale-free networks. Adv. Differ. Equ. 2019, 279 (2019)

24. Van den Driessche, P., Watmough, J.: Reproduction numbers and sub-threshold endemic equilibria for compartmental models of disease transmission. Math. Biosci. 180(1-2), 29-48 (2002)

25. Lajmanovich, A., Yorke, J.A.: A deterministic model for gonorrhea in a nonhomogeneous population. Math. Biosci. 28(3-4), 221-236 (1976)

26. Zhu, G., Fu, X., Chen, G.: Spreading dynamics and global stability of a generalized epidemic model on complex heterogeneous networks. Appl. Math. Model. 36(12), 5808-5817 (2012) 